\title{
Research on Teenagers' Static Balance Ability Based on Somatosensory Interaction
}

\author{
Qi Zhong1, Xiaoyan Wang'2, Wei Wang1* \\ ${ }^{1}$ Laboratory of Machine Learning and Cognition, School of Education Science, Nanjing Normal University, Nanjing, China \\ ${ }^{2}$ Linping the First Middle School, HangZhou, China \\ Email: *wan gwei5@njnu.edu.cn
}

How to cite this paper: Zhong, Q., Wang, X. Y., \& Wang, W. (2019). Research on Teenagers' Static Balance Ability Based on Somatosensory Interaction. Advances in Applied Sociology, 9, 15-31.

https://doi.org/10.4236/aasoci.2019.91002

Received: October 29, 2018

Accepted: January 5, 2019

Published: January 8, 2019

Copyright (c) 2019 by author(s) and Scientific Research Publishing Inc. This work is licensed under the Creative Commons Attribution International License (CC BY 4.0).

http://creativecommons.org/licenses/by/4.0/

\begin{abstract}
Good balance ability plays an important role in teenagers' physical development. Establishing a comprehensive evaluation system is conducive to promote the development of teenagers' balance ability test. In this study, based on the calculation of the center of mass, Kinect detects human body's joints to obtain 10 basic indicators of the static balance ability, calculate the weight coefficient and the variation coefficient by the variation coefficient method and calculate the coefficient values of different tasks and indexes, finally, combine coefficient linear with weight linear sum method to obtain comprehensive evaluation index formula of static balance ability. From the test items in the text, the weight coefficient of the single foot standing with closing eyes is relatively high $(29.23 \%)$, which had a greater influence on the final static balance ability assessment. In the test indicators, the weight coefficient of index in the swing speed of center of mass is relatively high (32.9\%), and that in swinging of the vertical axis of center of mass is also relatively high (30.0\%), taking great impact on the final test result. Finally, there is a comparison between the results of this study and experts' evaluation result, the correlation coefficient between the two $(|\mathrm{r}|>0.85, \mathrm{r}<0, \mathrm{p}<0.01)$ indicates that the two groups have a high negative correlation, and they match yet the evaluation system is more sensitive if two scores are normalized to the same interval.
\end{abstract}

\section{Keywords}

Balance Ability, Center of Mass, Variation Coefficient, Comprehensive Evaluation

\section{Introduction}

The balance ability of the body refers to the ability to maintain its own stability, 
including the ability to maintain a certain posture or keep balance by nerves and muscles when the body is physically affected, which is one of the important physiological functions of human body (Prado, Raso, Scharlach, \& Kasse, 2014). In terms of sports sciences, it is a skill considered within the scope of coordination which refers to the interaction of the central nervous system and the skeletal-muscular system in a mutual adjustment for the intended movement (Okudur \& Sanioğlu, 2012). There are two types of balance: static balance and dynamic balance. Static balance is the ability to achieve and maintain balance without moving; dynamic balance, while dynamic balance is the ability to move without losing balance or falling (Tanır, 2018). Here we focus on static balance stability in our study. It is really important for many sports, such as football, swimming, shooting, skiing and so on, especially gymnastics (Hrysomallis, 2011). The balance ability has been developing not only in the early childhood, but throughout adolescence (Nolan, Grigorenko, \& Thorstensson, 2005). In addition, some common diseases of teenagers and adults such as autism, hyperactivity disorder, Asperger's syndrome and others are closely related to the balance ability of human body (Cha \& Kim, 2016). Balance ability is one of the important components of the physical fitness of middle school students. However, it is easily neglected by physical education in the middle school which focuses on strength, speed and endurance of body. Therefore, it is necessary to detect, quantify and assess the balance ability of teenagers (Emery et al, 2005).

\subsection{Evaluation Index}

The main principle, in instrument evaluation, is to detect the related parameters of center of mass (COM) or center of gravity (COG) and center of pressure (COP), which can be used to reflect the balance stability of body and swing of body. COM is a centralized position of the human body, a hypothetical point weighted by the center of each body segment (Vlutters, Asseldonk, \& Kooij, 2016); COG is the point of gravity, which is often used in combination with COM (Boukhenous, Attari, \& Remram, 2013). The control of supporting surface of COM or COP reflects the stability of the human body. COP is the distribution center acting on all the forces on the supporting surface (Mukaino et al., 2016), so we develop static balance ability system based on COM.

During the process of maintaining the stable posture, the center of mass constantly swings forward, backward, left and right. So, the swing characteristics of the center of mass can be analyzed to reflect the level of human static balance ability (Wang \& Wang, 2014). The swing characteristics of center of mass include the swing type of center of mass, the swing length of center of mass, the swing speed of center of mass, the swing amplitude of center of mass and the swing area of center of mass, and the derived parameters such as Romberg rate (Mancini \& Horak, 2010). These parameters can objectively reflect the balance function of subjects. The subjects of this study are normal adolescents whose 
swing types of the center of mass are centered, so the swing type is ignored in the evaluation parameters of this study. Romberg rate is used to judge the cause of balance disorder which is suitable for patients with visual impairments, labyrinthine disturbance and spinal cord posterior cord disorder (Yun, 2005), but not for the subjects in this study. Above all, the swing parameters of center of mass in normal people are swing length, swing speed, swing amplitude and swing area of the center of mass (Lee, Cherng, \& Lin, 2004). The direction of movement in body and center of mass are based on the medio-lateral, the supero-inferior and the anterior-posterior.

\subsection{Evaluation Method}

Balance is a complex process involving many sensories, motor and bio-mechanical components in coordinated activities. For that reason, based on maintaining balance, provide sports performance by collecting effective strategies (Tanir, 2018). Standing balance is a very important part of balance control. The evaluation methods of that include clinical observation (Hedetoft \& Hyldegaard, 2015), scale evaluation (Wong, et al., 2015) and instrument evaluation. During the first two methods, graders mainly observe subjects' state of the completing the task intuitively, then give the artificial scores. They are too rough and subjective to quantify and refine the measurement results of balance ability and produce ceiling effect easily (Clark et al., 2010; Suzuki et al., 2018).

Segmental method in three-dimensional motion analysis system (Eng \& Winter, 1993; Hasan et al., 1996; Gullstrand et al., 2009) obtains the trajectory of left-right, front-back and vertical three directions of the COM through data acquisition of body segment calibration points. The dynamometer method is used to simulate the position of the COM by analyzing the change of the COP of the human body in three-dimensional space when it is placed on the ground. In addition, gravity line method, LPF method (Caron, 1997; Hof, 2005) and COP-COM (Winter et al., 1996; Corriveau, Hébert, Prince, \& Raîche, 2001) method based on inverted pendulum model are also commonly used. At present, the domestic balance detection instruments mainly use force measurements to obtain related parameters of the COP to reflect the balance ability, while the method based on the COM is seldom studied.

The $3 \mathrm{D}$ motion system can test the level of balance ability effectively and reliably, but the operation of this system is complex which needs to mark points with expensive equipment, thus it is not suitable for popularization and application in general schools.

Somatosensory interaction (Chen, Jun-Ze, \& Chang, 2013) is an action-induced interaction technology in intelligent interaction technology which uses limb movements, voices and facial expressions directly to interact with computer equipment or environment. The computer recognizes the user's limb movements and voices, and gives relevant feedback. In recent years, somatosensory interaction technology has played an important role in teaching, such as 
physical education to raise students' sense of participation and immersion (Kumara et al., 2015). The learning environment which combines somatosensory interaction with motion perception can fully mobilize learners' multi-sense and enhance human-computer interaction to help learners' health and greatly increase learners' interest during the class (Yang et al., 2014). It plays an important role in sports evaluation which improves the traditional testing methods and achieves quantitative evaluation objectively (Gieser, Metsis, \& Makedon, 2014).

Chakravarty et al. (Chakravarty et al., 2016) developed a new eigen vector based on curvature analysis algorithm to compute single limb stance (SLS) duration on the skeleton data and derived two parameters vibration-jitter and force per unit mass (FPUM) for each body part to assess postural stability during SLS. Clark et al. (Clark et al., 2012; Clark et al., 2015) assessed the concurrent validity of the Microsoft Kinect64 against a multiple-camera 3D motion analysis system during three postural control tests: 1) forward reach, 2) lateral reach, and 3) single-leg eyes-closed standing balance. These findings suggested that the Microsoft Kinect64 can validly assess kinematic strategies of postural control. These reaches focused on the parameters of joint points (hip joint, knee joint, ankle joint), however without calculating the center of mass or center of gravity which means less accuracy.

Y Yang et al. (Yang et al., 2014) found that the center of mass (COM) was calculated from the body's kinematic data acquired by the Kinect system and Optotrak Certus motion capture system. The position variability and average velocity of the COM in the horizontal plane were calculated and used to evaluate the subject's balance. These COM parameters from the two systems showed excellent and comparable test-retest reliability (intraclass correlation coefficient).

In LF Yeung's study (Yeung et al., 2014), they made a comparison between the Kinect system, a Vicon motion capture system and a force plate in four upright quiet standing tasks: 1) eyes open (EOn), 2) eyes closed (ECn), 3) eyes open standing on foam (EOf), and 4) eyes closed standing on foam (ECf) to calculate the balance ability. The experiment didn't calculate the change of the center of mass in the upper and lower directions that couldn't reflect the swing process of the body in space.

Lvet al. (Lv et al., 2016) compared results in center of mass between Kinect2 equipment, pressure tablet and balance board WII to verify the effectiveness of Kinect2 system and researched the effects of weight, height and BMI indexes on balance, but the test just dispersedly concluded that it lacks the establishment of a comprehensive evaluation system.

In view of above problems, this study designs a static balance ability evaluation project based on somatosensory interaction. Firstly, four testing tasks are determined according to the traditional testing methods and obtain the position of the center of mass by Kinect 2 equipment, Secondly, calculate the static ability 
evaluation indexes, calculate coefficient of variation and weight coefficient based on different tasks and indicators by variation coefficient method, and combines all indicators by linear weighted sum method to build a comprehensive model of multi-index and multi-task to estimate static balance ability. Finally, the effectiveness of this method and the expert method is tested to prove that the comprehensive indicators of this study are clearly distinguished and with better sensitivity.

\section{Method}

\subsection{Participants}

52 subjects, aged about 14 years old, from junior middle school in Nanjing Huayuan Middle School, were enrolled in the experiment and 26 subjects for each gender. The specific gender and age information is shown in Table 1.

\subsection{Experimental Setup}

The static balance ability test environment should be set up. A computer host was connected to the Kinect motion sensor device and the Kinect was placed on a 25 -inch (about $63 \mathrm{~cm}$ ) Kinect triangulation stand which was suitable for subjects' height.

The teenagers stood 2 meters away from the Kinect equipment and quietly stared at the target point paralleling to the line of sight with hands clenched and hanging on both sides of the waist. Four tasks: 20 seconds of double feet standing with opening eyes (DFOE), 20 seconds of double feet standing with closing eyes (DFCE), 10 seconds of single foot standing with opening eyes (SFOE), 10 seconds of single foot standing with closing eyes (SFCE). Children took a break between each two tests to maintain their best test status. Figure 1 shows a project process diagram during the test process.

This experiment used the second-generation somatosensory interaction device Kinect V2 provided by Microsoft Corp. The Kinect V2's infrared camera, depth camera and RGB camera were used to capture human's 3D motion datum and record them in the Kinect for Windows SDK, a development kit provided for users by Microsoft. This research mainly used the skeleton tracking technology to obtain 25 joints of the human body to provide initial datum for subsequent calculation of center of mass which are essential for related evaluation indicators and, finally, feedback test results through evaluation indicators. This evaluation system framework is mainly composed of human joint data acquisition module,

Table 1. 52 Subjects' basic information.

\begin{tabular}{cccc}
\hline & Total & Boy & Girl \\
\hline Number & 52 & 26 & 26 \\
Average age & 13.74 & 13.64 & 13.85 \\
\hline
\end{tabular}




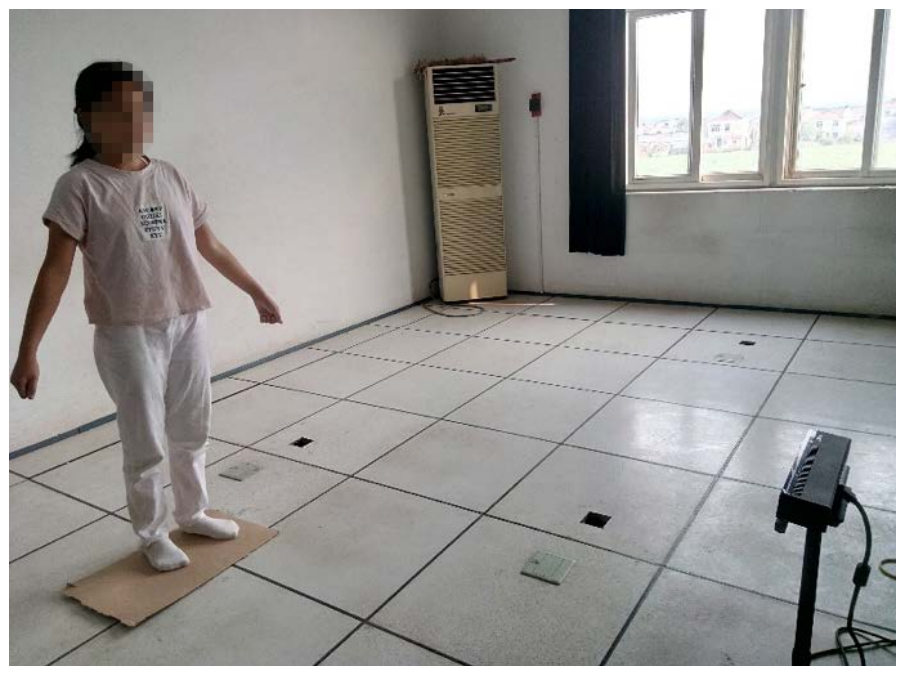

Figure 1. Process of static balance ability test.

center of mass position calculation module, evaluation index calculation module and test results feedback module. The preceding module provides data for the next module. The framework of the test system is shown in Figure 2, and the system interface is shown in Figure 3.

\subsection{Data}

In this section, we introduce how to get the data of the center of mass from the joint points and process the data of the center of mass to get 10 indexes of the swing length, the swing speed, the swing amplitude and the swing area of the center of mass. Detailed formulas are described as follows.

\subsubsection{Center of Mass}

The Kinect for Windows SDK 2.0 software development kit is compiled to write application program to obtain the data of 25 joints of human body. The data models of 19 joints are extracted and synthesized by coefficient synthesis method to calculate real-time center of mass position when human body swinging. The coefficients of the coefficient synthesis method in the experiment are selected from the data of the Germans, which are commonly used at present (Hong \& Ye, 1982). The formulas for calculating the center of mass are as follows:

$$
\begin{gathered}
G_{x}=\sum k_{i} * J x_{i} \\
G_{y}=\sum k_{i} * J y_{i} \\
G_{z}=\sum k_{i} * J z_{i}
\end{gathered}
$$

$G_{x}, G_{y}, G_{z}$ respectively represents the position of the center of mass in the left and right directions, vertical direction and forward and backward directions. $J_{X}$, $J y, J z$ respectively represents the position of joints in the left and right directions, vertical direction and forward and backward directions, and $K_{i}$ represents the corresponding coefficients of the i-th joint. 


\section{Static balance capability test system}

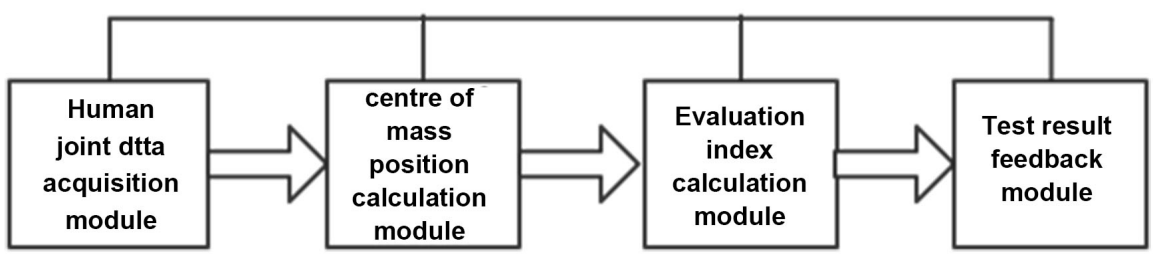

Figure 2. Frame diagram of test system.

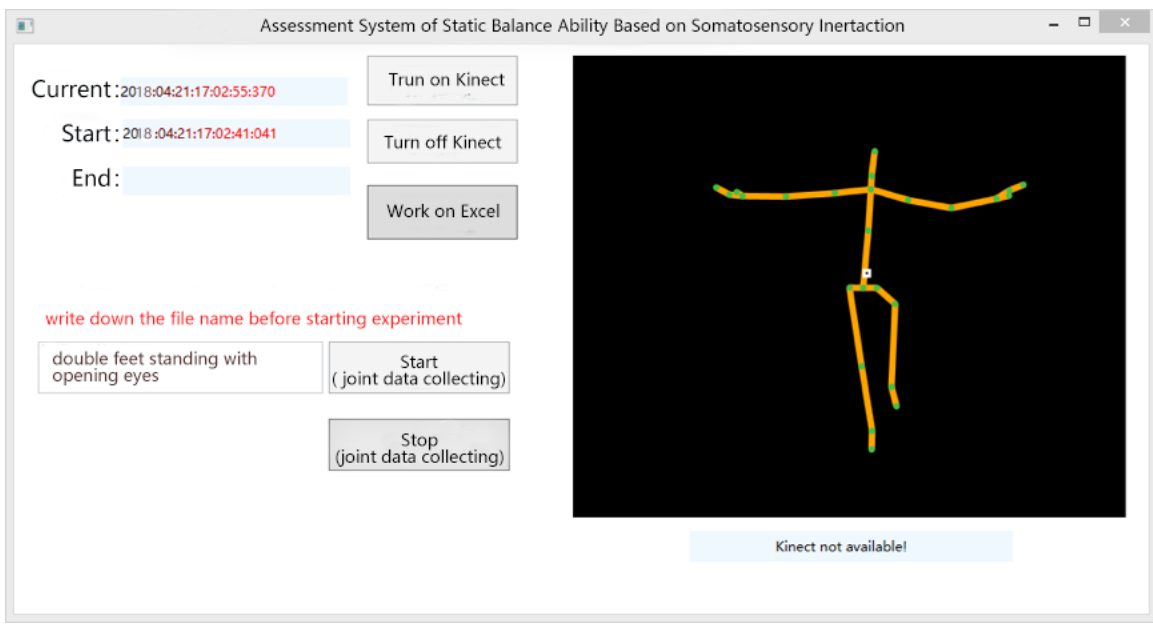

Figure 3. Operation diagram of test system.

\subsubsection{Static Capability Evaluation Indexes}

On the basis of calculating center of mass position, there are 10 static capability evaluation indexes needed to calculate, namely the medio-lateral swing length (MLSL), the supero-inferior swing length (SISL), the anterior-posterior swing length (APSL), the medio-lateral swing velocity (MLSV), the supero-inferior swing velocity (SISV), the anterior posterior swing velocity (APSV), the medio-lateral swing amplitude (MLSA), the supero-inferior swing amplitude (SISA), the anterior posterior swing amplitude (APSA), and the swing envelop area (SEA). These indexes give quantitative evaluation as the basic data of the static capability comprehensive evaluation system. The formulas are as follows:

The formulas for calculating the center of mass swing length are as follows.

$$
\begin{aligned}
M L S L & =\sum_{t=1}^{n-1}\left|G x_{t}-G x_{t+1}\right| \\
S I S L & =\sum_{t=1}^{n-1}\left|G y_{t}-G y_{t+1}\right| \\
A P S L & =\sum_{t=1}^{n-1}\left|G z_{t}-G z_{t+1}\right|
\end{aligned}
$$

$t$ represents the current frame, and $n$ represents the total number of frames during the test time.

The formulas for calculating the swing velocity of center of mass are as follows: 


$$
\begin{aligned}
M L S L & =M L S L / T \\
S I S V & =S I S L / T \\
A P S V & =A P S V / T
\end{aligned}
$$

T represents the test time.

The formulas for calculating the swing amplitude of center of mass are as follows:

$$
\begin{gathered}
M L S A=\left[\frac{1}{n} \sum_{t=1}^{n}\left(G x_{t}-\overline{G x}\right)^{2}\right]^{1 / 2} \\
S I S A=\left[\frac{1}{n} \sum_{t=1}^{n}\left(G y_{t}-\overline{G y}\right)^{2}\right]^{1 / 2} \\
A P S A=\left[\frac{1}{n} \sum_{t=1}^{n}\left(G z_{t}-\overline{G z}\right)^{2}\right]^{1 / 2}
\end{gathered}
$$

$\overline{G x} \quad \overline{G y} \quad \overline{G z}$ respectively represents the average position of the center of mass in the swinging process in the left and right directions, up and down directions, and forward and backward directions.

When calculating the swing area of the center of gravity, using the envelope area function conv hull in MATLAB 2014, the horizontal center of gravity scatter plot is drawn first, and then the peripheral center points are connected to form a convex polygon, as shown in Figure 4. Finally, the swing area of the horizontal center of gravity is returned.

\subsubsection{Synthetic Comprehensive Evaluation Index}

The above single item or single index test results are one-sided that can't reflect the static balance ability comprehensively, so the multi-index and multi-item evaluation system can be built to reflect the test level more comprehensively and objectively. The system is based on 10 basic evaluation indexes, and uses linear weighted sum method to synthesize many evaluation indexes into a final unique index. The different weighted coefficients can express the influence of different indexes on the final balance ability. Below is the formula:

$$
Y=\sum_{i=1}^{n} W_{i} X_{i}
$$

$X_{i}$ represents the i-th evaluation index, $W_{i}$ represents the weight coefficient corresponding to the $\mathrm{i}$-th evaluation index, $n$ is the number of evaluation indexe, $Y$ is the final comprehensive evaluation index.

The coefficient of variation method is used to determine the weight coefficient of each index, which is an objective weighting method (Brown, 2011). Coefficient of variation can eliminate the influence of measurement scale and dimension of different evaluation index on balance ability, reflect the dispersion of index, the discrimination of index and the difference of each index value (Reed, Lynn, \& Meade, 2002). Below is the formula of coefficient of variation: 


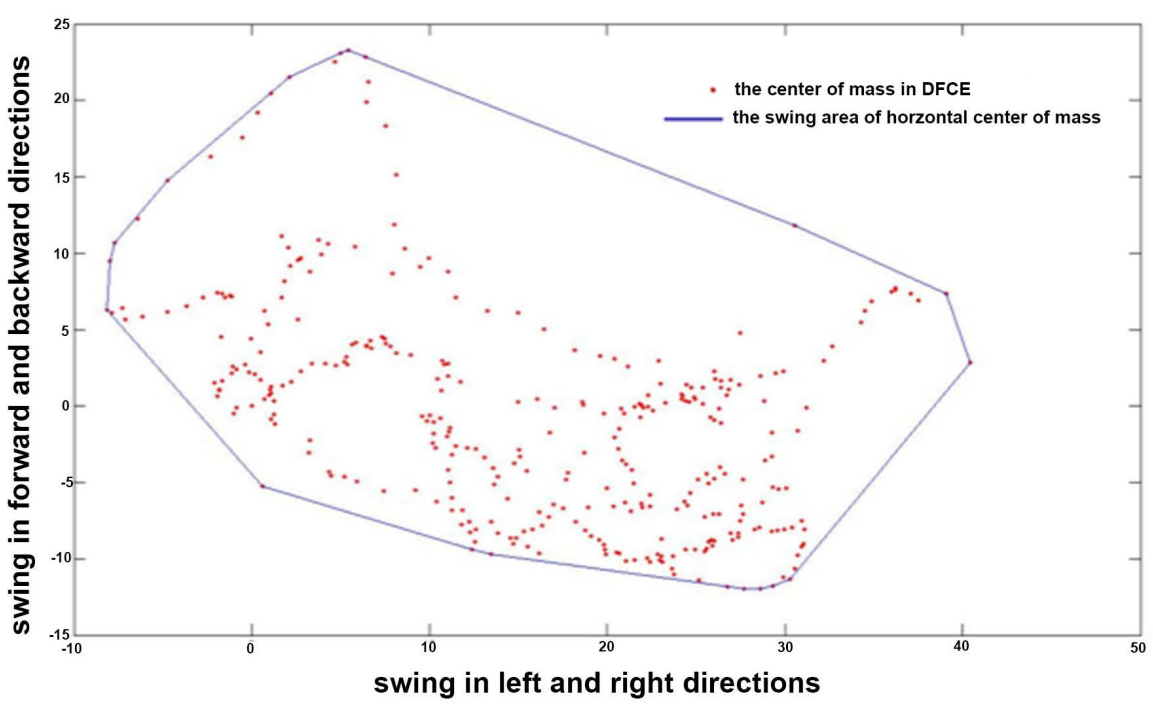

Figure 4. Horizontal center of gravity swinging area.

$$
V_{i}=\frac{\sigma_{i}}{\bar{x}_{i}}(i=1,2, \cdots, n)
$$

$V_{i}$ represents the coefficient of variation of the $i$-th indicator, also known as the standard deviation coefficient, $\sigma_{i}$ represents the standard deviation of the $i$-th indicator, $\bar{x}_{i}$ represents the average of the $i$-th indicator.

The weight calculation method of each index is as follow. The greater the coefficient of variation $\left(V_{i}\right)$, the stronger the ability to distinguish the $i$-th index, and the greater the influence on the static balance ability.

$$
w_{i}=\frac{V_{i}}{\sum_{i=1}^{n} V_{i}}
$$

$w_{i}$ represents different weight coefficients of different indexes.

\section{Result}

A total of 52 subjects completed 4 test items, 10 evaluation indexes to complete 2080 test results. The average test results of the 4 test items are as shown in $\mathrm{Ta}$ ble 2. Table 2 shows that the 10 evaluation indexes of the same test item are different, so it is difficult to reflect the overall level of static balance ability according to the single index.

In order to classify and weigh the data more detailed, we calculated the weight coefficients from different items and different indexes. Firstly, we calculated the weight coefficients in different items. The weight coefficients are compared in Tables 3-6.

SD represents Standard Deviation, VC represents variation coefficients, WC represents weight coefficient.

From above tables, we can see that the weight coefficient of SFEC has the largest proportion as DFEO takes the least (DFEO 21.51\%, DFEC 22.67\%, SFEO $26.6 \%$, SFEC $29.23 \%$ ). Therefore, as the difficulty of the test items increasing, the 
Table 2. Average test result of the 4 test items.

\begin{tabular}{ccccc}
\hline items & DFOE & DFCE & SFOE & SFCE \\
\hline index & 53.48 & 52.00 & 170.38 & 288.28 \\
APSL & 163.03 & 187.83 & 453.27 & 357.10 \\
SISL & 103.34 & 112.24 & 368.92 & 355.56 \\
$M L S V$ & 2.67 & 2.60 & 17.05 & 24.52 \\
APSV & 8.16 & 9.40 & 45.37 & 37.99 \\
SISV & 5.17 & 5.61 & 36.92 & 37.42 \\
$M L S A$ & 2.30 & 2.28 & 4.94 & 12.11 \\
APSA & 1.14 & 1.18 & 4.62 & 7.01 \\
SISA & 4.77 & 4.81 & 8.63 & 13.21 \\
SEA & 102.74 & 118.56 & 477.88 & 1924.90 \\
\hline
\end{tabular}

Table 3. Weight coefficient of 10 evaluation indexes in DFOE items.

\begin{tabular}{ccccc}
\hline Index & Average & SD & CV & WC \\
\hline$M L S L_{1}$ & 53.48 & 53.36 & 0.997 & 0.029 \\
$A P S L_{1}$ & 163.03 & 165.65 & 1.016 & 0.030 \\
$S I S L_{1}$ & 103.34 & 52.75 & 0.510 & 0.015 \\
$M L S V_{1}$ & 2.68 & 2.67 & 0.998 & 0.129 \\
$A P S V_{1}$ & 8.16 & 8.29 & 1.016 & 0.030 \\
$S I S V_{1}$ & 5.17 & 2.64 & 0.510 & 0.015 \\
$M L S A_{1}$ & 2.30 & 1.16 & 0.508 & 0.015 \\
$A P S A_{1}$ & 1.14 & 0.61 & 0.528 & 0.016 \\
$S I S A_{1}$ & 4.77 & 2.48 & 0.521 & 0.015 \\
$S E A_{1}$ & 102.74 & 70.07 & 0.682 & 0.020 \\
$D F E O$ & & & 7.286 & 0.215 \\
\hline
\end{tabular}

SD represents Standard Deviation, VC represents variation coefficients, WC represents weight coefficient.

Table 4. Weight coefficient of 10 evaluation indexes in DFCE items.

\begin{tabular}{ccccc}
\hline Index & Average & SD & CV & WC \\
\hline$M L S L_{2}$ & 52.00 & 31.57 & 0.607 & 0.018 \\
APSL $_{2}$ & 187.84 & 256.56 & 1.366 & 0.040 \\
SISL $_{2}$ & 112.24 & 78.14 & 0.696 & 0.021 \\
$M L S V_{2}$ & 2.60 & 1.58 & 0.607 & 0.018 \\
$A P S V_{2}$ & 9.40 & 12.84 & 1.366 & 0.040 \\
SISV $_{2}$ & 5.62 & 3.91 & 0.696 & 0.021 \\
MLSA & 2.29 & 1.24 & 0.544 & 0.016 \\
APSA & 1.18 & 0.63 & 0.536 & 0.016 \\
$S I S A_{2}$ & 4.81 & 2.29 & 0.476 & 0.014 \\
$S E A_{2}$ & 118.56 & 93.10 & 0.785 & 0.023 \\
DFEC & & & 7.679 & 0.226 \\
\hline
\end{tabular}


Table 5. Weight coefficient of 10 evaluation indexes in SFOE items.

\begin{tabular}{ccccc}
\hline Index & Average & SD & CV & WC \\
\hline MLSL $_{3}$ & 170.38 & 152.97 & 0.898 & 0.027 \\
APSL $_{3}$ & 453.27 & 578.28 & 1.276 & 0.038 \\
SISL $_{3}$ & 368.92 & 423.00 & 1.147 & 0.034 \\
$M L S V_{3}$ & 17.05 & 15.31 & 0.898 & 0.027 \\
APSV $_{3}$ & 45.37 & 57.89 & 1.276 & 0.038 \\
ISV $_{3}$ & 36.93 & 42.34 & 1.147 & 0.034 \\
MLSA $_{3}$ & 4.95 & 1.87 & 0.378 & 0.011 \\
APSA $_{3}$ & 4.62 & 3.253 & 0.704 & 0.021 \\
SISA $_{3}$ & 8.64 & 4.95 & 0.573 & 0.017 \\
SEA $_{3}$ & 477.89 & 341.93 & 0.716 & 0.021 \\
SFEO & & & 9.010 & 0.266 \\
\hline
\end{tabular}

Table 6. Weight coefficient of 10 evaluation indexes in SFCE items.

\begin{tabular}{ccccc}
\hline Index & Average & SD & CV & WC \\
\hline MLSL $_{4}$ & 288.26 & 139.22 & 0.610 & 0.018 \\
APSL $_{4}$ & 357.10 & 445.02 & 1.246 & 0.037 \\
SISL $_{4}$ & 355.56 & 466.63 & 1.312 & 0.039 \\
$M L S V_{4}$ & 24.56 & 18.09 & 0.737 & 0.022 \\
APSV $_{4}$ & 37.99 & 46.85 & 1.233 & 0.036 \\
SISV $_{4}$ & 37.42 & 47.91 & 1.280 & 0.038 \\
MLSA $_{4}$ & 12.11 & 10.46 & 0.864 & 0.025 \\
APSA & & 4.77 & 0.681 & 0.200 \\
SISA $_{4}$ & 7.01 & 6.99 & 0.529 & 0.016 \\
$S E A_{4}$ & 13.22 & 2174.70 & 1.410 & 0.042 \\
$S F E C$ & 1924.90 & & 9.903 & 0.292 \\
\hline
\end{tabular}

proportion of the indexes will increase, and more difficult tests can reflect the static balance level of the subjects better.

In addition, we calculate the weight coefficients from different indexes. Table 7 is a comparison of weight coefficients in different indexes.

According to these indexes, we can find that weight coefficient in SEA contains the largest proportion above all $(\mathrm{WC}=0.169)$, which means it will take more influence on final balance ability result and will reflect balance ability better. However, this in SISA is only 0.052, the least one, which means it has little influence on final comprehensive result. From the different aspects of indexes in Table 8, the weight coefficient of the center of gravity swing velocity (SV) is the largest $(32.9 \%)$, which has a great influence on the static balance ability. From the different directions of the indexes in Table 9, weight coefficient of the 
Table 7. Weight coefficients in 10 indexes.

\begin{tabular}{ccccc}
\hline Index & Average & SD & CV & WC \\
\hline MLSL & 126.03 & 131.58 & 1.044 & 0.074 \\
APSL & 290.31 & 410.82 & 1.415 & 0.105 \\
SISL & 235.02 & 341.02 & 1.451 & 0.104 \\
$M L S V$ & 11.71 & 15.18 & 1.296 & 0.093 \\
APSV & 25.23 & 41.28 & 1.636 & 0.117 \\
SISV & 21.28 & 35.59 & 1.674 & 0.119 \\
$M L S A$ & 5.41 & 6.69 & 1.236 & 0.088 \\
$A P S A$ & 3.48 & 3.81 & 1.094 & 0.078 \\
SISA & 7.86 & 5.74 & 0.730 & 0.052 \\
SEA & 656.01 & 1552.80 & 2.360 & 0.169 \\
\hline
\end{tabular}

Table 8. Weight coefficient in the different aspects of indexes.

\begin{tabular}{ccccc}
\hline Index & SL & SV & SA & SEA \\
\hline WC & 0.283 & 0.329 & 0.218 & 0.169 \\
\hline
\end{tabular}

Table 9. Weight coefficient in the different directions of indexes.

\begin{tabular}{ccccc}
\hline Index & ML & AP & SI & SEA \\
\hline WC & 0.255 & 0.300 & 0.275 & 0.169 \\
\hline
\end{tabular}

vertical axis swing is the largest $(30.0 \%)$, with the greatest impact on static balance ability.

Finally, we establish a comprehensive evaluation index of static balance ability through the weighted linear sum method above these weight coefficients from different static balance ability evaluation items and indexes. The formula can be expressed as:

$$
\begin{aligned}
& S=0.074 \times\left(0.215 \times M L S L_{1}+0.226 \times M L S L_{2}+0.266 M L S L_{3}+0.292 \times M L S L_{4}\right) \\
& +0.105 \times\left(0.215 \times A P S L_{1}+0.226 \times A P S L_{2}+0.266 \times A P S L_{3}+0.292 \times A P S L_{4}\right)+ \\
& 0.104 \times\left(0.215 \times S I S L_{1}+0.226 \times S I S L_{2}+0.266 S I S L_{3}+0.292 \times S I S L_{4}\right)+0.093 \times \\
& \left(0.215 \times M L S V_{1}+0.226 \times M L S V_{2}+0.266 M L S V_{3}+0.292 \times M L S V_{4}\right)+0.117 \times \\
& \left(0.215 \times A P S V_{1}+0.226 \times A P S V_{2}+0.266 \times A P S V_{3}+0.292 \times A P S V_{4}\right)+0.119 \times \\
& \left(0.215 \times S I S V_{1}+0.226 \times S I S V_{2}+0.266 S I S V_{3}+0.292 \times S I S V_{4}\right)+0.088 \times(0.215 \times \\
& \left.M L S A_{1}+0.226 \times M L S A_{2}+0.266 M L S A_{3}+0.292 \times M L S A_{4}\right)+0.078 \times(0.215 \times \\
& \left.A P S A_{1}+0.226 \times A P S A_{2}+0.266 \times A P S A_{3}+0.292 \times A P S A_{4}\right)+0.052 \times(0.215 \times \\
& \left.S I S A_{1}+0.226 \times S I S A_{2}+0.266 S I S A_{3}+0.292 \times S I S A_{4}\right)+0.169 \times\left(0.215 \times S E A_{1}+\right. \\
& \left.0.226 \times S E A_{2}+0.266 S E A_{3}+0.292 \times S E A_{4}\right) .
\end{aligned}
$$

\section{Discussion}

To test the validity of the comprehensive evaluation indicator model, we compare the comprehensive evaluation index value according to model with the ex- 
pert score of adolescents given by professional physical teachers indegree of consistency. We make further calculations based on the average, standard deviation, coefficient of variation and so on for the expert scores to facilitate the comparison of the two scores. The results of Pearson correlation analysis of comprehensive evaluation score and expert score are shown in Table 10.

Pearson correlation coefficient of the two groups' results showed that there is a very significant negative correlation between the two results $(\mathrm{r}=0.908, \mathrm{P}<$ $0.01)$. There is highly correlation between comprehensive evaluation score and expert score $(|\mathrm{r}|>0.85$ indicates that there is a high correlation), meaning that the comprehensive index value is effective and this model can reflect balance ability well. And a negative correlation is showed between comprehensive evaluation score and expert score $(r<0)$, indicating that a person with good static balance ability will get high expert score and low comprehensive evaluation score which show completely opposite changes. The coefficient of variation of the system is 0.824 which is obviously greater than that of the expert's score (VC $=0.101$ ), showing that the comprehensive system is more obvious than that of the expert's score in distinguishing degree, which means the distinguishing ability of system is better. Therefore, when the subjects' static balance ability is poor, the swing characteristic value of the subjects' center of mass is large resulting in the large value of system evaluation index.

The dimension of the evaluation index and the expert score are different so that the two scores can't be compared at the same interval. It is necessary to normalize the evaluation index score and the expert score to the same interval. At the same time, because of the negative correlation between the two scores, the comprehensive evaluation index score is linearly transformed and plotted in order to directly comparing the two groups of score results, which is showed in Figure 5. The horizontal axis represents the number of subjects, and the longitudinal axis is the comprehensive score of static balance ability.

The trend of the two test groups is basically the same with the high consistency between $[70,100]$. When the scores are lower than 70 , the score of comprehensive evaluation indexes is much lower than that of experts. It is probably because the experts have the lowest score criteria for these adolescents that all the subjects scores are greater than 60 . Whereas the testing range of comprehensive system is more extensive without the lowest score to distinguish the better and the worse subjects more sensitively.

The coefficients in final evaluation indexes show that the item in single foot standing with opening eyes has the greatest weight coefficient which makes the

Table 10. Results of Pearson correlation analysis of comprehensive evaluation score and expert score.

\begin{tabular}{cccccc}
\hline Comprehensive score & Average & SD & VC & r & p \\
\hline$C S$ & 201.05 & 165.74 & 0.824 & -0.908 & 0.00 \\
$E S$ & 85.32 & 8.67 & 0.101 & & \\
\hline
\end{tabular}




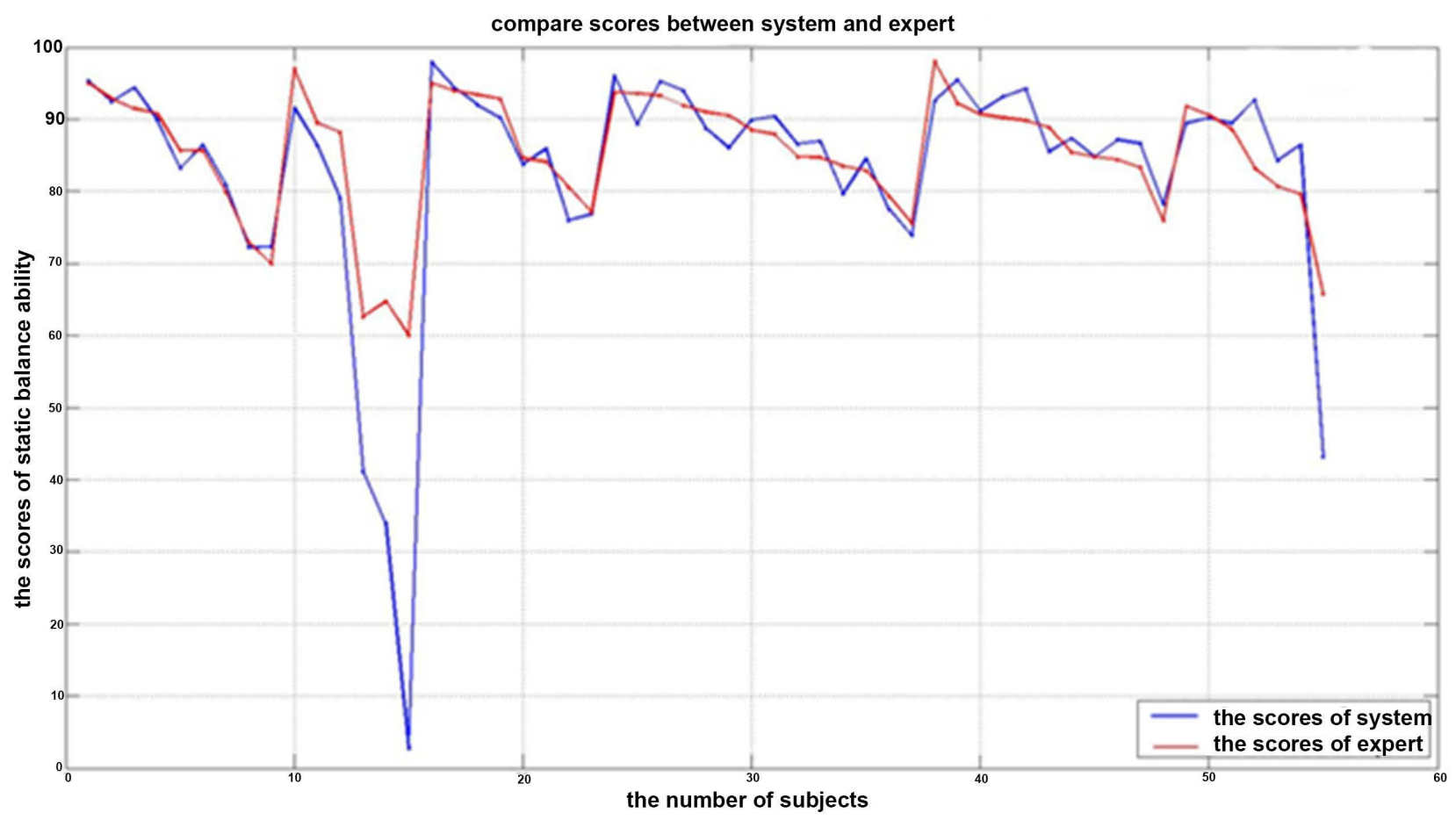

Figure 5. Comparison of comprehensive evaluation index scores with expert scores.

greatest contribution to the evaluation score, because this testing item is quite difficult to finish, the index value varies greatly, the coefficient of variation is larger and the weight coefficient is also larger. The weight coefficient of the swing area of the horizontal center of mass is the largest index, which makes a great contribution to final result, because the swing area of the horizontal center of mass represents the potential level of the balance ability, the subjects' static balance ability varies greatly, the coefficient of variation is bigger and the weight coefficient is bigger.

After the comprehensive evaluation index score and the expert score are normalized to the same interval, the scores in these two groups are basically consistent. The comprehensive evaluation index is more sensitive which can distinguish the difference of static balance ability better and can produce more comprehensive test results for its synthetic in 10 evaluation indexes among every four test items.

\section{Conclusion}

In this study, we calculate 10 basic evaluation indexes of static balance ability, such as swing speed, swing amplitude and swing speed according to center of mass and determine weight coefficients of different items and different evaluation indexes by using coefficient of variation method. Then weighted linear sum method is used to combine all above data into final evaluation indexes, and this comprehensive test system for static balance ability of Kinect is finished. The results of the evaluation system, to achieve process evaluation and comprehen- 
sive results, have better accuracy in good static balance of the subjects, and more obvious distinction and better sensitivity. Compared with the three-dimensional sports system, this system is easy to operate with low cost equipment, which is suitable for schools in balance ability measurements, especially in selecting elite athletes for Gymnastics. It will be more widely and in-depth used in balance ability measurement. This study is only aiming at some teenagers' static balance ability to establish test indexes. However, the subjects are limited. Next step is to improve the data of the test system to make the system more scientific.

\section{Conflicts of Interest}

The authors declare no conflicts of interest regarding the publication of this paper.

\section{References}

Boukhenous, S., Attari, M., \& Remram, Y. (2013). An Easy Platform for Postural Balance Analysis by the Evaluation of Instantaneous Center of Gravity. APCBEE Procedia, 7, 11-15. https://doi.org/10.1016/j.apcbee.2013.08.004

Brown, C. E. (2011). Coefficient of Variation. Encyclopedia of Statistical Sciences, 94, 94.

Caron, O. (1997). Estimating the Centre of Gravity of the Body on the Basis of the Centre of Pressure in Standing Posture. Journal of Biomechanics, 30, 1169-1171. https://doi.org/10.1016/S0021-9290(97)00094-8

Cha, H. G., \& Kim, M. K. (2016). Effects of Mental Practice on Normal Adult Balance Ability. Journal of Physical Therapy Science, 28, 2041-2043.

Chakravarty, K., Suman, S., Bhowmick, B., Sinha, A., \& Das, A. (2016). Quantification of Balance in Single Limb Stance Using Kinect. IEEE International Conference on Acoustics, Speech and Signal Processing (pp. 854-858). Piscataway, New Jersey, US: IEEE. https://doi.org/10.1109/ICASSP.2016.7471796

Chen, D., Jun-Ze, W., \& Chang, Q. U. (2013). Kinect Somatosensory Interaction Technology and Its Application in Medical Rehabilitation (Review). Chinese Journal of Rehabilitation Theory and Practice.

Clark, R. A., Bryant, A. L., Pua, Y., Mccrory, P., Bennell, K., \& Hunt, M. (2010). Validity and Reliability of the Nintendowii Balance Board for Assessment of Standing Balance. Gait \& Posture, 31, 310. https://doi.org/10.1016/j.gaitpost.2009.11.012

Clark, R. A., Pua, Y. H., Fortin, K., Ritchie, C., Webster, K. E., Denehy, L., et al. (2012). Validity of the Microsoftkinect for Assessment of Postural Control. Gait \& Posture, 36, 372-377. https://doi.org/10.1016/j.gaitpost.2012.03.033

Clark, R. A., Pua, Y. H., Oliveira, C. C., Bower, K. J., Thilarajah, S., Mcgaw, R., et al. (2015). Reliability and Concurrent Validity of the Microsoftxbox One Kinect for Assessment of Standing Balance and Postural Control. Gait \& Posture, 42, 210-213. https://doi.org/10.1016/j.gaitpost.2015.03.005

Corriveau, H., Hébert, R., Prince, F., \& Raîche, M. (2001). Postural Control in the Elderly: An Analysis of Test-Retest and Interrater Reliability of the Cop-Com Variable. Archives of Physical Medicine \& Rehabilitation, 82, 80-85. https://doi.org/10.1053/apmr.2001.18678

Emery, C. A., Cassidy, J. D., Klassen, T. P., Rosychuk, R. J., \& Rowe, B. B. (2005). Development of a Clinical Static and Dynamic Standing Balance Measurement Tool Appropriate for Use in Adolescents. Physical Therapy, 85, 502-514. 
Eng, J., \& Winter, D. (1993). Estimations of the Horizontal Displacement of the Total Body Centre of Mass: Considerations during Standing Activities. Gait and Posture, 1, 141-144. https://doi.org/10.1016/0966-6362(93)90055-6

Gieser, S. N., Metsis, V., \& Makedon, F. (2014). Quantitative Evaluation of the Kinect Skeleton Tracker for Physical Rehabilitation Exercises.

Gullstrand, L., Halvorsen, K., Tinmark, F., Eriksson, M., \& Nilsson, J. (2009). Measurements of Vertical Displacement in Running, a Methodological Comparison. Gait \& Posture, 30, 71-75. https://doi.org/10.1016/j.gaitpost.2009.03.001

Hasan, S. S., Robin, D. W., Szurkus, D. C., Ashmead, D. H., Peterson, S. W., \& Shiavi, R. G. (1996). Simultaneous Measurement of Body Center of Pressure and Center of Gravity during Upright Stance. Part Ii: Amplitude and Frequency Data. Gait \& Posture, 4, 1-10. https://doi.org/10.1016/0966-6362(95)01030-0

Hedetoft, M., \& Hyldegaard, O. (2015). Postural Stability in Patients with Decompression Sickness Evaluated by Means of Quantitative Romberg Testing. Undersea \& Hyperbaric Medicine, 42, 389-398.

Hof, A. L. (2005). Comparison of Three Methods to Estimate the Center of Mass during Balance Assessment. Journal of Biomechanics, 38, 2134-2135.

https://doi.org/10.1016/j.jbiomech.2005.03.029

Hong, Y., \& Ye, L. (1982). The Review and Research of Human Body Center of Gravity. Journal of Beijing Sport University, No. 3, 39-47.

Hrysomallis, C. (2011). Balance Ability and Athletic Performance. Sports Medicine, 41, 221-232. https://doi.org/10.2165/11538560-000000000-00000

Kumara, W. G. C. W., Wattanachote, K., Battulga, B., Shih, T. K., \& Hwang, W. Y. (2015). A Kinect-Based Assessment System for Smart Classroom. International Journal of Distance Education Technologies, 13, 34-53.

https://doi.org/10.4018/IJDET.2015040103

Lee, H. Y., Cherng, R. J., \& Lin, C. H. (2004). Development of a Virtual Reality Environment for Somatosensory and Perceptual Stimulation in the Balance Assessment of Children. Computers in Biology \& Medicine, 34, 719-733. https://doi.org/10.1016/j.compbiomed.2003.10.004

Lv, Z., Penades, V., Blasco, S., Chirivella, J., \& Gagliardo, P. (2016). Evaluation of Kinect2 Based Balance Measurement. Neurocomputing, 208, 290-298. https://doi.org/10.1016/j.neucom.2015.12.128

Mancini, M., \& Horak, F. B. (2010). The Relevance of Clinical Balance Assessment Tools to Differentiate Balance Deficits. European Journal of Physical and Rehabilitation Medicine, 46, 239-248.

Mukaino, M., Matsuda, F., Sassa, R., Ohtsuka, K., Kumazawa, N., Tsuchiyama, K. et al. (2016). Objective Measurement of Dynamic Balance Function by the Simultaneous Measurement of the Center of Gravity (cog) and Center of Pressure (cop).

Nolan, L., Grigorenko, A., \& Thorstensson, A. (2005). Balance Control: Sex and Age Differences in 9- to 16-Year-Olds. Developmental Medicine \& Child Neurology, 47, 449-454. https://doi.org/10.1017/S0012162205000873

Okudur, A., \& Sanioğlu, A. (2012). The Relationship between Balance and Agility Performance in Tennis Players Aged 12. Selçuk University Journal of Physical and Sport Science, 14, 165-170.

Prado, E., Raso, V., Scharlach, R., \& Kasse, C. (2014). Hatha Yoga on Body Balance. International Journal of Yoga, 7, 133-137. https://doi.org/10.4103/0973-6131.133893

Reed, G. F., Lynn, F., \& Meade, B. D. (2002). Use of Coefficient of Variation in Assessing 
Variability of Quantitative Assays. Clinical and Diagnostic Laboratory Immunology, 9, 1235-1239. https://doi.org/10.1128/CDLI.9.6.1235-1239.2002

Suzuki, Y., Yatoh, S., Suzuki, H., Tanabe, Y., Shimizu, Y., Hada, Y. et al. (2018). Age-Dependent Changes in Dynamic Standing-Balance Ability Evaluated, Quantitatively Using a Stabilometer. Journal of Physical Therapy Science, 30, 86-91. https://doi.org/10.1589/jpts.30.86

Tanır, H. (2018). The Effect of Balance and Stability Workouts on the Development of Static and Dynamic Balance in 10-12-Year-Old Soccer Players. Journal of Education and Training Studies, 9, 132-135. https://doi.org/10.11114/jets.v6i9.3499

Vlutters, M., Asseldonk, E. H. F. V., \& Kooij, H. V. D. (2016). Center of Mass Velocity Based Predictions in Balance Recovery Following Pelvis Perturbations during Human Walking. Journal of Experimental Biology, 219, 1514-1523. https://doi.org/10.1242/jeb.129338

Wang, L., \& Wang, W. (2014). Research on Kinect Attention Distance Assessment and Training System Based on Net Platform. Modernization of Education, 2.

Winter, D. A., Prince, F., Frank, J. S., Powell, C., \& Zabjek, K. F. (1996). Unified Theory Regarding $\mathrm{a} / \mathrm{p}$ and $\mathrm{m} / \mathrm{l}$ Balance in Quiet Stance. Journal of Neurophysiology, 75, 2334-2343. https://doi.org/10.1152/jn.1996.75.6.2334

Wong, C. K., Chen, C. C., Blackwell, W., Rahal, R., \& Benoy, S. (2015). Balance Ability Measured with the Berg Balance Scale: A Determinant of Fall History in Community-Dwelling Adults with Leg Amputation. Journal of Rehabilitation Medicine, 47, 80-86. https://doi.org/10.2340/16501977-1882

Yang, H. Y., Zhang, H., Xu, W., Zhang, P. J., \& Xu, L. M. (2014) The Application of KINECT Motion Sensing Technology in Game-Oriented Study. iJET, 9, 59-63. https://doi.org/10.3991/ijet.v9i2.3282

Yang, Y., Pu, F., Li, Y., Li, S., Fan, Y., \& Li, D. (2014). Reliability and Validity of Kinectrgb-d Sensor for Assessing Standing Balance. IEEE Sensors Journal, 14, 1633-1638. https://doi.org/10.1109/JSEN.2013.2296509

Yeung, L. F., Cheng, K. C., Fong, C. H., Lee, W. C. C., \& Tong, K. Y. (2014). Evaluation of the Microsoft Kinect as a Clinical Assessment Tool of Body Sway. Gait \& Posture, 40, 532-538. https://doi.org/10.1016/j.gaitpost.2014.06.012

Yun, X. P. (2005). Rehabilitation Therapy Evaluation. Huaxia Publishing. 